\title{
Beschaffung im Management-Fokus
}

Das Aufgabenspektrum der an der Klinikbeschaffung Beteiligten wandelt sich - und zwar gravierend. War der Klinikeinkäufer früher vor allem für die Abwicklung von Bestellungen und der Logistiker für die Verteilung der Waren zuständig, so steht jetzt die strategische Beschaffung im Fokus. Dadurch rücken Einkauf und Logistik enger zusammen und werden zu einer wichtigen Schnittstelle im Versorgungssystem des Krankenhauses. Der Einkäufer ist inzwischen Berater für die Ärzte und die Krankenhausleitung, er soll eine Übersicht über den Markt und gute Beziehungen zu den Lieferanten haben und zudem strategisch und mit Weitblick arbeiten, um auch wirtschaftlich gute Zahlen vorlegen zu können. Und natürlich sollen möglichst alle Prozesse digitalisiert ablaufen. Auch im Klinikeinkauf sucht man jetzt also die eierlegende Wollmilchsau. Wunsch und Wirklichkeit klaffen derzeit in vielen Kliniken aber noch weit auseinander.

Mit einem medienübergreifenden Dreiklang aus Magazin, Newsletter und OnlinePortal wollen wir dabei unterstützen, die Menschen mitzunehmen auf dem Weg von einer Bestellabteilung zu einem digitalisierten strategischen Beschaffungsmanagement. Mit Klinik Einkauf wollen wir allen Beteiligten das erforderliche Rüstzeug geben, um ihre zentrale Rolle im Beschaffungsprozess voll auszufüllen.

Mit diesem Heft halten Sie die erste Ausgabe des Magazins Klinik Einkauf in den Händen. Darin werden Sie Fachbeiträge, Praxisbeispiele und aktuelle Produkt- und Brancheninformationen sowie Informationen zu den rechtlichen und politischen Rahmenbedingungen rund um die Themen Beschaffungsmanagement und Logistik finden. Unsere hochkarätig besetzten Gremien Redaktionsbeirat und Expertenpanel werden Sie durch die große
Themenvielfalt aus Wissenschaft, Praxis und Industrie leiten.

Das Magazin ist Teil einer crossmedialen Informationsplattform für Klinikeinkäufer, Logistiker und Entscheider im Beschaffungsprozess und ergänzt das Klinik Einkauf Online-Portal und den Klinik Einkauf Newsletter. Gleichzeitig ist Klinik Einkauf eine Plattform, die zum Austausch einladen soll. In unserem Blog unter www.klinik-einkauf.de bringen wir aktuelle Themen auf den Tisch und laden zur Diskussion ein. Gleichzeitig sind wir auf den Social-Media-Kanälen wie Twitter und Xing unterwegs. So machen wir Klinik Einkauf zu einer umfassenden Informations- und Dialogplattform, über die Klinikeinkäufer miteinander in Kontakt treten und erfahren können, was ihre Kollegen gerade bewegt.

Ich wünsche Ihnen eine kurzweilige Lektüre und freue mich, wenn Sie bei unserem neuen Online-Portal Klinik Einkauf reinschauen und auf den Seiten stöbern.

Herzliche Grüße

\section{Carolin Freye}

Programmplanung Gesundheitswirtschaft Thieme Gruppe

- Besuchen Sie unser neues Online-Portal www.klinik-einkauf.de

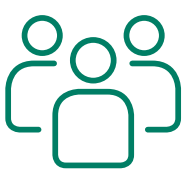

Beiräte aus Wissenschaft und Praxis

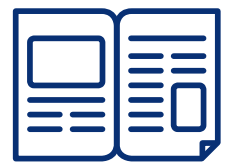

$3500+$ Auflage

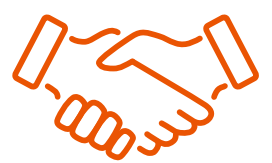

Partner \& Sponsoren

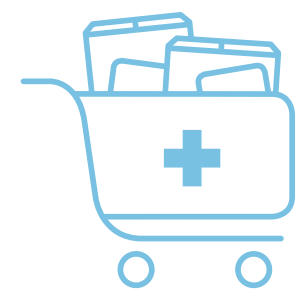

